\title{
Elevated Plasma Level of the Glycolysis Byproduct Methylglyoxal on Admission Is an Independent Biomarker of Mortality in ICU COVID-19 Patients
}

\section{Fadhel A. Alomar}

Imam Abdulrahman Bin Faisal University

Marai N. Alshakhs

Dammam Medical Complex

Salah Abohelaika

Qatif Central Hospital, Ministry of Health

Hassan M. Almarzouk

Dammam Medical Complex

Mohammed Almualim

Qatif Central Hospital, Ministry of Health

Amein K. Al-Ali

Imam Abdulrahman Bin Faisal University

Fahad Al-Muhanna

Imam Abdulrahman Bin Faisal University

Mohammed F. Alomar

Imam Abdulrahman Bin Faisal University

Mousa J. Alhaddad

Dammam Medical Complex

Mohammed S. Almulaify

Dammam Medical Complex

Faisal S. Alessa

Dammam Medical Complex

Ahmed S. Alsalman

Dammam Medical Complex

Ahmed Alaswad

Qatif Central Hospital, Ministry of Health

Sean R. Bidasee

University of Nebraska Medical Center

Hassan Alsaad

Imam Abdulrahman Bin Faisal University

Rudaynah A Alali 
Imam Abdulrahman Bin Faisal University

\section{Mona H. AlSheikh}

Imam Abdulrahman Bin Faisal University

\section{Mohammed S. Akhtar}

Imam Abdulrahman Bin Faisal University

\section{Mohammed Al Mohaini}

Abdulaziz University for Health Sciences

\section{Abdulkhaliq J. Alsalman}

Northern Border University

Hussain Alturaifi

King Fahad Hospital Hofuf

Keshore R. Bidasee ( $\sim$ kbidasee@unmc.edu )

University of Nebraska Medical Center

\section{Research Article}

Keywords: Elevated Plasma, Glycolysis Byproduct, Mortality in ICU

Posted Date: December 15th, 2021

DOI: https://doi.org/10.21203/rs.3.rs-1075237/v1

License: (c) (1) This work is licensed under a Creative Commons Attribution 4.0 International License. Read Full License 


\section{Abstract}

Biomarkers to identify ICU COVID-19 patients at high risk for mortality are urgently needed for therapeutic care and management. In this study we found that plasma levels of the glycolysis byproduct methylglyoxal $(\mathrm{MG})$ were 4.4 -fold and 1.7 -fold higher $(\mathrm{P}<0.0001)$ upon ICU admission in patients that later died $(n=34)$ compared to uninfected controls $(n=30)$ and those survived $(n=31)$, respectively. The increase in MG was inversely correlated with glutathione $\left(r^{2}=-0.63\right)$ and the MG-glutathione degrading glyoxalase- $1\left(r^{2}=-0.50\right)$, and positively correlated with the inflammation markers, SSAO $\left(r^{2}=0.52\right)$, TNF-a $\left(r^{2}=0.41\right), \mathrm{IL}-1 \mathrm{~b}\left(r^{2}=0.25\right), \mathrm{CRP}\left(r^{2}=0.26\right)$ and age $\left(r^{2}=0.20\right)$. Logistic regression analysis provides evidence of a significant relationship between the elevated MG upon admission into ICU and death $(P<0.0001)$, with $42 \%$ of the death variability explained. From these data we conclude that elevated plasma MG on admission is a novel independent biomarker that predicts ICU COVID-19 patients at high risk of mortality.

\section{Introduction}

The novel coronavirus, termed severe acute respiratory syndrome coronavirus 2 (SARS-CoV-2), has claimed the lives of 5 million deaths worldwide ${ }^{1}$. As of as of Nov ${ }^{3 r d}, 2021$ more than 247 million individuals have been infected ${ }^{2}$. Although the majority of individuals infected with SARS-CoV-2 remain asymptomatic or develop mild fever, cough, muscle weakness, headache, sore throat, diarrhea, and loss of taste and smell, about $10 \%$ of individuals develop acute respiratory distress (ARDS) requiring intensive care unit (ICU) hospitalization ${ }^{3-6}$. Advanced age, pre-existing medical conditions including cardiovascular diseases, cancers, diabetes mellitus and those that were not vaccinated are at higher risk of developing ARDS requiring ICU hospitalization ${ }^{7-10}$. In fact, individuals with chronic diabetes mellitus (DM) that are infected with the SARS-CoV-2 virus are three times more likely to die from coronavirus disease 2019 (COVID-19) compared to infected individuals without $\mathrm{DM}^{11-13}$. Many ICU patients that survived may also develop post-COVID syndrome, referred to as long COVID. Symptoms of post-COVID syndrome include persistent immunosuppression, pulmonary, cardiac, and vascular fibrosis that will negatively impact their quality of life ${ }^{14,15}$.

Because the number of COVID-19 patients requiring ICU treatment is extremely large (1 in 10) and exceeds the capacity of most hospitals, biomarkers are currently used to assist health care workers to stratify and triage patients for management and care. These biomarkers fall into five broad categories: (1) hematological/coagulation markers including platelet to lymphocyte ratios, ferritin and D-dimer, (2) inflammation markers including serum lactate dehydrogenase (LDH), C-reactive protein (CRP), procalcitonin, interleukins (IL), IL-1b, IL-2, IL-6, IL-8, IL-10, IL-17, CXCL10/IP-10, and tumor necrosis factor (TNF-a), (3) cardiac dysfunction markers including troponin I (cTnl), N-terminal pro brain natriuretic peptide (NT-proBNP), a-hydroxybutyrate dehydrogenase (a-HBDH) and creatine kinase-myocardial band (CK-MB), (4) liver function markers including aspartate aminotransferase (AST), alanine aminotransferase (ALT), total bilirubin, gamma-glutamyl transferase (GGT), and serum albumin, and (5) 
renal function markers including glomerular filtration rate and blood urea nitrogen ${ }^{16-18}$. In a metaanalysis of thirty-two studies, Malik and colleagues found significant associations between blood lymphopenia, thrombocytopenia, elevated CRP, procalcitonin, lactate dehydrogenase, D-dimer, ALT, and AST with adverse COVID-19 outcomes. Four of these thirty-two studies reported ICU death as an endpoint ${ }^{19}$. In another report, Narvel and colleagues pointed out that although currently used biomarkers are useful for stratifying/ triaging patients for ICU admittance, they are not specific for COVID-1920, and are mainly downstream consequences of cellular changes that occur following infection. Biomarkers associated with early replication of SARS-CoV-2 could provide objective and unbiased information to health care professionals to better manage and predict outcomes of ICU COVID-19 patients. These early markers may also provide insights into novel treatment strategies to blunt complications resulting from SARS-CoV2 infection.

The SARS-CoV-2 virus infects human cells expressing the angiotensin-converting enzyme 2 (ACE2) including type 1 and type 2 pneumocytes, vascular endothelial cells, epithelial cells, and peripheral blood mononuclear cells (PBMCs) ${ }^{21-23}$. Like most viruses, after entry the SARS-CoV-2 virus reprograms host cellular metabolism to obtain the building blocks needed for its own replication and survival. These metabolic changes include upregulating glycolysis, amino acids and lipid synthesis, and impairment in mitochondrial functions ${ }^{24-26}$. Ajaz et al., reported an increase in extracellular acidification rate (ECAR) of PBMCs isolated from COVID-19 patients, suggestive in part of an increase in glycolysis flux ${ }^{27}$. Several glycolysis pathway genes are also upregulated in PBMCs from COVID-19 patients, including alphaenolase, glyceraldehyde 3-phosphate dehydrogenase, pyruvate kinase M1/2 PKM, phosphomutase 2, hexokinase, lactate dehydrogenase A and triosephosphate isomerase 1 (TPI1) ${ }^{28}$. Studies have also found reduced basal and maximal respiration and reduced spare respiratory capacity in monocytes and peripheral blood mononuclear cells (PBMCs) indicative of impaired mitochondrial function ${ }^{29,30}$.

In addition to producing ATP and pyruvate, glycolysis also generates the cytotoxic byproduct, methylglyoxal (MG) from the interconversion of glyceraldehyde 3-phosphate and dihydroxyacetone phosphate via TPI ${ }^{31}$. In healthy individuals, plasma and tissue MG levels are kept low by the actions of dual-enzyme glyoxalase system that rapidly degrades the hemithioacetal formed between MG and glutathione (MG-GSH) into D-lactic acid and glutathione ${ }^{32,33}$. Expression of the rate-limiting enzyme that degrades the MG-GSH hemithioacetal, glyoxalase-I (EC4.4.1.5, Glo1) is negatively regulated by the inflammation, hypoxia, and oxidative stress ${ }^{34-37}$, conditions seen in COVID-19 patients. Thus, an increase in MG production via glycolysis coupled with impaired degradation from reduced expression of Glo1 will lead to accumulation of MG in blood and tissues of COVID-19 patients. At supraphysiologic levels, MG will disrupt the function of vascular endothelial cells resulting in microvascular leakage, clot and tissue fibrosis $^{38-41}$. Supraphysiological levels of MG will also potentiate inflammation and oxidative stress by activating NF-KB and the NLR family pyrin domain containing 3 (NRLP3) inflammasome, and the activities of complex I and III of the electron transport chain in mitochondria ${ }^{42,43}$. 
To this end, a cross-sectional study was conducted to (i) determine if MG levels are elevated in plasma of ICU COVID-19 patients shortly after admittance in Qatif Central Hospital; and (ii) determine if elevation in plasma MG on admittance is predictive of death. Glutathione and glyoxalase-1 (Glo1) levels were also measured to gain insight into mechanisms that contribute to MG accumulation. The inflammation markers, semicarbazide-sensitive amine oxidase (SSAO); the soluble form of soluble vascular adhesion protein 1 (VAP-1), TNF-a, IL-1b and CRP were also measured as their levels are increased in part from MG accumulation.

\section{Results}

\section{Characterization of patients used in study}

The general characteristics, medication history and blood profile of these patients are shown in Tables 1 and 2. The sixty-five ICU patients were separated into those with diabetes mellitus (DM) and those without diabetes mellitus (non-DM) (Table 1). There were no significant differences in the mean age and weights of patients (Table 1). Uninfected individuals were slightly younger and weighed less than ICU patients. A higher percentage (41\%) of non-DM were on antiviral regimens. Blood glucose, creatinine, ALT and AST were higher in ICU patients compared to uninfected individuals (Table 2). Other blood parameters between the groups are listed in Table 2. Of the thirty-four non-DM patients in ICU, sixteen died (47\%), and seventeen of thirty-one DM patients died (55\%), see (Table 1). 
Table 1

Demographic data of the uninfected controls and ICU-COVID-19 patients

\begin{tabular}{|c|c|c|c|c|}
\hline & & Control & COVID-19 & \\
\hline & & $(n=30)$ & $\begin{array}{l}\text { Non-DM } \\
(n=34)\end{array}$ & $\begin{array}{l}\text { DM } \\
(n=31)\end{array}$ \\
\hline \multirow[t]{2}{*}{ Sex } & Male & 22 & 18 & 21 \\
\hline & Female & 8 & 16 & 10 \\
\hline Age, year Median (range) & & $\begin{array}{l}43(27- \\
73)\end{array}$ & $46(30-80)$ & $54(28-81)$ \\
\hline $\begin{array}{l}\text { Weight kg Median } \\
\text { (range) }\end{array}$ & & $70(60-95)$ & $80(55-115)$ & $\begin{array}{l}80(59- \\
120)\end{array}$ \\
\hline \multirow[t]{15}{*}{ Treatments (\%) } & Antivirals & & 41 & 12 \\
\hline & - Lopinavir/Ritonavir & & & \\
\hline & • Ribavirin & & & \\
\hline & - Interferon & & & \\
\hline & - Favipiravir & & & \\
\hline & Antibiotics & & 85 & 80 \\
\hline & - Ceftriaxone & & & \\
\hline & - Azithromycin & & & \\
\hline & - Linezolid & & & \\
\hline & - Vancomycin & & & \\
\hline & • Tazocin & & & \\
\hline & • Meropenem & & & \\
\hline & Steroids & & 96 & 84 \\
\hline & - Dexamethasone & & & \\
\hline & - Methylprednisolone & & & \\
\hline \multirow[t]{4}{*}{ Comorbidities (\%) } & Respiratory disease & & 11 & 8 \\
\hline & Hypertension & & 30 & 76 \\
\hline & $\begin{array}{l}\text { Ischemic heart disease } \\
\text { IHD }\end{array}$ & & 15 & 8 \\
\hline & Hyperlipidemia & & 4 & 12 \\
\hline
\end{tabular}




\begin{tabular}{|llll|}
\hline & Control & COVID-19 & \\
\hline Renal failure & 11 & 24 \\
\hline Died n (\%) & $16(47 \%)$ & $17(55 \%)$ \\
\hline
\end{tabular}


Table 2

Analytes in blood of uninfected controls and ICU COVID-19 patients

\begin{tabular}{|c|c|c|c|}
\hline & Control & COVID-19 & \\
\hline & $n=30$ & $\begin{array}{l}\text { Non-diabetic } \\
\mathrm{n}=34\end{array}$ & $\begin{array}{l}\text { Diabetic } \\
\mathrm{n}=\mathbf{3 1}\end{array}$ \\
\hline $\begin{array}{l}\text { Glucose random } \\
\text { (3.3-9.99 mmol/L) }\end{array}$ & $5.4 \pm 1.2$ & $8.1 \pm 2.7 \uparrow$ & $14.5 \pm 7.0 \uparrow$ \\
\hline $\begin{array}{l}\text { Hemoglobin } \\
(13-17 \mathrm{~g} / \mathrm{dL})\end{array}$ & $13.9 \pm 1.1$ & $11 \pm 2.4 \downarrow$ & $10.5 \pm 1.8 \downarrow$ \\
\hline $\begin{array}{l}\text { Hematocrit } \\
(49-54 \%)\end{array}$ & $45.1 \pm 3.2$ & $35.1 \pm 7.4 \downarrow$ & $33.7 \pm 7.5 \downarrow$ \\
\hline $\begin{array}{l}\text { WBC } \\
\left(4-10 \times 10^{3} / \mu \mathrm{L}\right)\end{array}$ & $5.1 \pm 1.3$ & $9.2 \pm 3.7 \uparrow$ & $10.9 \pm 4.6 \uparrow$ \\
\hline $\begin{array}{l}\text { RBC } \\
\left(4.5-5.5 \times 10^{6} / \mu \mathrm{L}\right)\end{array}$ & $5.7 \pm 0.8$ & $4.4 \pm 0.9$ & $4.4 \pm 0.9$ \\
\hline $\begin{array}{l}\text { Platelet } \\
\left(150-430 \times 10^{3} / \mu \mathrm{L}\right)\end{array}$ & $249.3 \pm 64.5$ & $286.9 \pm 113.3$ & $274.5 \pm 139.7$ \\
\hline $\begin{array}{l}\text { Sodium } \\
(135-153 \mathrm{mmol} / \mathrm{L})\end{array}$ & $141.1 \pm 1.7$ & $139.9 \pm 7.1$ & $140.6 \pm 6.9$ \\
\hline $\begin{array}{l}\text { Potassium } \\
(3.5-5.3 \mathrm{mmol} / \mathrm{L})\end{array}$ & $4.3 \pm 0.3$ & $4.2 \pm 0.7$ & $4.4 \pm 0.5$ \\
\hline $\begin{array}{l}\text { Chloride } \\
\text { (96-106 mEq/L) }\end{array}$ & $100.9 \pm 1.5$ & $102.6 \pm 7.7$ & $102.4 \pm 6.7$ \\
\hline $\begin{array}{l}\text { Calcium } \\
(2.1-2.55 \mathrm{mmol} / \mathrm{L})\end{array}$ & $2.4 \pm 0.1$ & $2.1 \pm 0.2$ & $2.1 \pm 0.2$ \\
\hline $\begin{array}{l}\text { Phosphorus } \\
\text { (0.8-1.6 mmol/L) }\end{array}$ & $1.1 \pm 0.2$ & $1.2 \pm 0.4$ & $1.3 \pm 0.5$ \\
\hline $\begin{array}{l}\text { Magnesium } \\
(0.7-1.0 \mathrm{mmol} / \mathrm{L})\end{array}$ & & $0.9 \pm 0.1$ & $0.9 \pm 0.1$ \\
\hline
\end{tabular}




\begin{tabular}{|c|c|c|c|}
\hline & Control & COVID-19 & \\
\hline $\begin{array}{l}\text { Albumin } \\
\text { (35-52 g/dL) }\end{array}$ & $47.1 \pm 2.4$ & $31.8 \pm 5.2 \downarrow$ & $30.3 \pm 4.6 \downarrow$ \\
\hline Urea (2.5-6.4 mmol/L) & $4.7 \pm 1.4$ & $9 \pm 6.9 \uparrow$ & $12.6 \pm 6.4 \uparrow$ \\
\hline $\begin{array}{l}\text { Creatinine } \\
(53-106 \mu \mathrm{mol} / \mathrm{L})\end{array}$ & $84.5 \pm 19.8$ & $89.8 \pm 58.8$ & $131.5 \pm 72.3 \uparrow$ \\
\hline $\begin{array}{l}\text { Total bilirubin } \\
(0-20 \mu \mathrm{mol} / \mathrm{L})\end{array}$ & $12.1 \pm 12.6$ & $22.8 \pm 57.1 \uparrow$ & $11.7 \pm 9.3$ \\
\hline Alanine aminotransferase (10-50 U/L) & $23.5 \pm 15.1$ & $41.6 \pm 33.6$ & $148.2 \pm 446.8 \uparrow$ \\
\hline Aspartate aminotransferase (0-38 U/L) & $22.5 \pm 14.2$ & $49.2 \pm 39.9 \uparrow$ & $316.2 \pm 1331.5 \uparrow$ \\
\hline $\begin{array}{l}\text { Lactate dehydrogenase } \\
(81-230 \mathrm{U} / \mathrm{L})\end{array}$ & & $611.4 \pm 655.9 \uparrow$ & $586 \pm 772.2 \uparrow$ \\
\hline $\begin{array}{l}\text { Creatine phosphokinase } \\
(38-308 \mathrm{U} / \mathrm{L})\end{array}$ & $160.5 \pm 213.8$ & $346.3 \pm 826.3 \uparrow$ & $357.4 \pm 393.9 \uparrow$ \\
\hline Creatine phosphokinase-MB (7-25 U/L) & $15.6 \pm 3.9$ & $31.7 \pm 30.2 \uparrow$ & $33.6 \pm 23.8 \uparrow$ \\
\hline PT (11.5-15.5 sec) & 14.8 & $15.4 \pm 4.7$ & $15.1 \pm 3.4$ \\
\hline PTT (26.4-36 sec) & 37.8 & $34.5 \pm 7.1$ & $31.9 \pm 6.6$ \\
\hline INR (0.85-1.15) & 1.1 & $1.2 \pm 0.5$ & $1.1 \pm 0.3$ \\
\hline Lactate $(0.5-2.2 \mathrm{mmol} / \mathrm{L})$ & & $1.7 \pm 0.6$ & $2.2 \pm 1.5$ \\
\hline Ferritin $(30-400 \mu \mathrm{g} / \mathrm{L})$ & & $1618 \pm 1600 \uparrow$ & $1216 \pm 1083 \uparrow$ \\
\hline $\begin{array}{l}\text { Cholesterol } \\
(1.3-5.2 \mathrm{mmol} / \mathrm{L})\end{array}$ & & $4.2 \pm 2.4$ & $3.2 \pm 1.0$ \\
\hline $\begin{array}{l}\text { Triglycerides } \\
(0.34-1.69 \mathrm{mmol} / \mathrm{L})\end{array}$ & & $2.6 \pm 1.5$ & $1.9 \pm 1.0$ \\
\hline $\begin{array}{l}\text { HDL-Cholesterol } \\
(1.03-1.55 \mathrm{mmol} / \mathrm{L})\end{array}$ & & $0.8 \pm 0.3$ & $0.8 \pm 0.3$ \\
\hline $\begin{array}{l}\text { LDL-Cholesterol } \\
(1.3-2.6 \mathrm{mmol} / \mathrm{L})\end{array}$ & & $2.2 \pm 1.1$ & $1.7 \pm 0.9$ \\
\hline
\end{tabular}




\section{Plasma levels of MG, glutathione, Glo1, SSAO, TNF-a, IL-1b, CRP in all ICU COVID-19 patients}

Plasma levels of MG, glutathione, and the inflammation markers SSAO, TNF-a, IL-1b, CRP, but not Glo1, were significantly higher in all ICU patients compared to uninfected individuals (Figs. 1A-F). When ICU patients were separated into those that were discharged and those that died, MG was significantly higher (77\%), and glutathione (44\%) and Glo1 (43\%) were significantly lower in plasma in patients that died (Figs. 2A-2C). There were no significant differences in plasma levels of SSAO, TNF-a, IL-1b, and CRP between patients that died and survived (Figs. 2D-2G).

\section{Plasma levels of MG, glutathione, Glo1, SSAO, TNF-a, IL-1b, CRP in non-DM and DM patients}

Studies have shown that patients with DM are at higher risk for severe COVID-19 outcomes compared with non-DM patients. ${ }^{11-13}$ In our study, there were no significant differences in plasma levels of MG, glutathione, Glo1, TNF-a, and CRP in non-DM and DM ICU patients upon ICU admittance (Figs. 3A, 3C, 3E, 4C, and 4G). However, SSAO and IL-1b were significantly higher in plasma of DM patients upon admission into ICU (Figs. 4A and 4E). Interestingly, in non-DM and DM patients that died, plasma MG was higher and glutathione and Glo1 were lower compared to patients that survived (Figs. 3B, 3D and 3F). There were no significant differences in plasma levels of the inflammation markers SSAO, TNF-a, IL-1b and CRP between DM and non-DM patients that died and survived (Figs. 4B, 4D, 4F and 4H).

\section{Mortality in ICU patients with low and high plasma MG}

Plasma MG on ICU admittance was then used to further separate patients that survived and died. MG in uninfected controls ( $495 \mu \mathrm{g} / \mathrm{mL}$ HSA-MG) was set as reference. Patients that survived were categorized into two group, those with MG up to 2-fold higher than uninfected controls were considered as low MG patients, and those with plasma MG 2-3-fold higher than control were considered as moderate MG patients (Fig. 5A). All patients with low plasma MG upon admittance $(11 / 65,17 \%)$, were discharged from ICU. the Median time to discharge was 7 days, range of 5-22 days. Patients $(21 / 65,32 \%)$ with moderate MG levels that survived had a mean time to discharge of 8.5 days, with a range of 3-32 days. Patients that dies were also categorized into two group. The first group contained patients with MG less than 4fold than uninfected controls (moderate MG patients), and the second group contained patients with MG $>$ 4-fold than uninfected controls (high MG patients), Fig. 5A. In the moderate MG group, time to death $(13 / 65,20 \%)$ was 25 days, range $14-31$ days and in the high MG group $(21 / 65,32 \%)$, the median time to death was 14 days with a range of 9-26 days. Figs. 5B shows Kaplan-Meier curves for all patients that died and separating them into non-DM and DM (Figs. 5C and 5D). These data show that the higher the plasma MG the earlier the onset of death, regardless of whether the patient has DM or not. 
A forward selection logistic regression model indicates evidence of a significant relationship between MG and COVID-19 patients (logistic regression chi-square $=24.90, \mathrm{df}=1, \mathrm{P}<0.0001$ ). Dead patients were found to have significantly higher MG $(P<0.0001)$ compared to their survived counterparts. The model correctly predicted $67 \%$ of death cases and explained $42 \%$ of the variability.

\section{Correlations between MG, glutathione, Glo1, SSAO, TNF-a, IL-1b and CRP COVID-19 patients that died}

Next, we investigated relationships between plasma MG levels in patients that died and glutathione, Glo1, SSAO, TNF-a, IL-1b, CRP and age to gain insights into causes and consequences of MG accumulation. In this study, strong inverse correlations were found between plasma MG and glutathione $\left(r^{2}=-0.62\right)$ and Glo1 ( $\left.r^{2}=-0.50\right)$, Figs. $6 \mathrm{~A}$ and $6 \mathrm{~B}$. Positive correlations between plasma MG and the inflammation markers, SSAO $\left(r^{2}=0.52\right)$ and TNF-a $\left(r^{2}=0.41\right)$ were also observed, Figs. $6 \mathrm{C}$ and $6 \mathrm{D}$. Weaker correlations were observed with IL-1b $\left(r^{2}=0.25\right)$, CRP $\left(r^{2}=0.26\right)$ and age $\left(r^{2}=0.20\right)$, Figs. $6 \mathrm{E}, 6 \mathrm{~F}$, and $6 \mathrm{G}$.

\section{Discussion}

About $10 \%$ of people infected with the SARS-CoV-2 virus develop severe respiratory distress syndrome (COVID-19) requiring hospitalization and intensive care ${ }^{4-6}$. About $50 \%$ of all ICU patients with COVID-19 also died from the disease ${ }^{44}$. To date, biomarkers to predict which ICU COVID-19 patients are at high risk of mortality are insufficient ${ }^{1,5,16-20}$. We reasoned those metabolic byproducts closely associated with SARS-CoV-2 replication could afford additional and unbiased information about the severity of infection as well as shed light into novel therapeutic approaches for treatment of ICU COVID-19 patients and to blunt post-COVID 19 complications.

The principal finding of the present study is that high plasma levels of the glycolysis byproduct MG upon admission into ICU is a predictor of death in COVID-19 patients. In this cross-sectional study, plasma samples were obtained from sixty-five COVID-19 patients shortly after admission into intensive care units (ICU) and the patients were monitored until they were discharged or died. All COVID-19 patients admitted into the ICU had 3.5-fold higher level of MG compared to that in uninfected controls. The plasma levels of glutathione were also reduced 2.9-fold. The rate-limiting MG degrading enzyme Glo1 did not change. The inflammation markers originating from separate pathways; SSAO from increased expression of the inflammation-induced protein vascular adhesion protein-1 ${ }^{45}$, TNF-a resulting from activation of nuclear factor kappa-light-chain-enhancer of activated B cells (NF-kB), IL-1b resulting from activation of the inflammasome ${ }^{42,46}$, and CRP resulting from generalized inflammation ${ }^{47}$, were increased 5.2-, 3.3-, 2.0-, and 7.7-fold respectively, compared to uninfected control.

After categorizing the ICU patients, we found patients that died had $77 \%$ higher plasma MG compared to those who survived. This elevation in plasma MG in patients that died was accompanied by $44 \%$ and $43 \%$ reductions in both glutathione and Glo1, respectively, suggesting that degradation of MG was being compromised. Interestingly, ICU COVID-19 patients that died had similar plasma levels of SSAO, TNF-a, IL- 
$1 \mathrm{~b}$ and CRP to those who survived, but significantly higher levels than that in uninfected controls. As expected, when MG levels are high, TNF-a and IL-1b are elevated along with the inflammation-induced proteins SSAO, and CRP. It should be mentioned that the parent molecule from which soluble SSAO is derived; membrane-bound vascular adhesion protein-1 (VAP-1), also generates MG from the deamination of aminoacetone $e^{45}$.

Patients with DM are at elevated risk of severe respiratory and adverse outcomes including death following SARS-CoV2 infection compared to non-DM patients ${ }^{7,11-13}$. This prompted us to separate our ICU cohorts into non-DM and DM with further subdivision into those that survived and those that died. MG levels were $70 \%$ and $68 \%$ higher in non-DM and DM patients that died compared to patients that survived. Plasma glutathione levels were also reduced by $41 \%$ and $46 \%$, and Glo1 levels were reduced by $37 \%$ and $49 \%$ in both non-DM and DM patients that died compared to those who survived, consistent with the notion that degradation of MG is being compromised in non-DM and DM patients that died.To gain further insight into the relationship between plasma MG levels and death, the logistic regression analysis revealed evidence of a significant relationship between MG and COVID-19 patients that died. Our model also correctly predicted $67 \%$ of death cases in ICU COVID-19 patients and explained $42 \%$ of the death variability. To the best of our knowledge, these data are the first to show that elevated plasma MG in ICU COVID-19 patients upon admission is predictive of death. Although, plasma levels of SSAO, TNF-a, IL-1b and CRP were significantly higher in COVID-19 patients than uninfected controls, they were similar in ICU COVID-19 patients that survived and died.

In this study, ICU patients that died had reduced plasma levels of glutathione and Glo1. To date, the underlying causes for reduction of plasma glutathione levels in COVID-19 patients remain poorly defined. However, we suspect that this could be arising in part from the increase in oxidative stress reported in COVID-19 patients. In an earlier report Horowitz and colleagues showed that oral and intravenous glutathione and the glutathione precursors ( $\mathrm{N}$-acetyl-cysteine) attenuated activation of NF-kB, cytokine storm syndrome and respiratory distress syndrome seen in COVID-19 patients with pneumonia. It is likely that intravenous glutathione and the glutathione precursors ( $\mathrm{N}$-acetyl-cysteine) administration could also be lowering MG levels by forming MG-glutathione hemiacetal. Glutathione is synthesized in two sequential reactions. In the first reaction, y-glutamylcysteine ligase (GCL; EC 6.3.2.2) converts I-glutamate and I-cysteine into Y-glutamylcysteine, and in the second reaction, glutathione synthetase (GSS; EC 6.3.2.3) adds glycine to $y$-glutamylcysteine to form glutathione ${ }^{38,48,49}$. To date, there is a paucity of information on expression and activities of $\gamma$-glutamylcysteine ligase and glutathione synthetase in the context of COVID-19 in the literature.

The underlying causes for the reduction plasma Glo1 levels in plasma of COVID-19 patients also remain poorly defined. The promoter region of human GLO1 has a functionally operative antioxidant response element (ARE) ${ }^{34-36}$. Under non-stress conditions, the antioxidant transcription factor nuclear factor erythroid 2-related factor 2 (Nrf2) binds to the ARE region of GLO1 to induce its expression ${ }^{50}$. NF-KB antagonizes the binding of Nrf2 to ARE to inhibit Glo1 expression. Since activation of NF-KB is 
upregulated in COVID-19 patients ${ }^{51}$, this could account in part for the reduction plasma Glo1. Hypoxiainducible factor 1a (HIF-1a) whose expression is upregulated in COVID-19 patients binds to the ARE and suppresses Glo1 expression ${ }^{34-36}$. HIF-1a is one of the two subunits of the heterodimeric transcription factor that regulates cellular and systemic adaptive responses to low oxygen (hypoxia) $37,43,52,53$. When oxygen delivery is compromised, as is the case with respiratory distress syndrome and ischemia in COVID-19, HIF-1 a escapes degradation, allowing it to migrate to the nucleus and induce transcription of HIF-1a target genes, including those involved in glycolysis and erythropoiesis ${ }^{37}$. The increase in glycolysis and suppression of Glo1 expression by HIF-1a inadvertently leads to an increase in MG. To the best of our knowledge, there is no published literature on linking HIF-1a upregulation and elevation in MG in COVID-19 patients.

This study is not without limitations. We have shown that MG is increased in plasma of ICU patients that died with COVID-19 and this increase in MG is associated with reductions in glutathione and Glo1, indicating that MG degradation is being compromised. We have also shown increased expression of SSAO, which is known to generate MG from the deamination of aminoacetone, a substrate that is derived from the breakdown of glycine and threonine. However additional work is needed to characterize this mechanism. Additional in vitro studies are also needed to quantitate changes in glycolysis and MG production in SARS-CoV2-infected cells.

In conclusion, the present study shows for the first time that accumulation of the cytotoxic glycolysis metabolite MG is increased in plasma from ICU COVID-19 patients. Dead patients were found to have significantly higher plasma levels of MG compared to their survived counterparts. A forward selection logistic regression model indicates evidence of a significant relationship between MG plasma levels and COVID-19 severity and mortality. The model correctly predicted $67 \%$ of death cases and explained $42 \%$ of the death variability. From these data we conclude that elevated plasma MG on admission could be a novel independent biomarker that predicts ICU COVID-19 patients at high risk of mortality.

\section{Materials And Methods Study Participants}

This study was approved by the ethical committee of Imam Abdulrahman Bin Faisal University, Al Kubar (IRB \# 2020-05-184), and of Qatif Central Hospital (QCH-SREC0229/2020). COVID-19 patients admitted to the ICU in Qatif Central Hospital between October 2020 to Feb 2021 were recruited into this study after informed consent was taken. All ICU patients had respiratory rate $\geq 30$ beats $/ \mathrm{min}$; blood oxygen saturation $\leq 93 \%$ at rest; arterial oxygen partial pressure $\left(\mathrm{PaO}_{2}\right)$ / oxygen concentration $\left(\mathrm{FiO}_{2}\right)$ ratio <300; lung infiltrates $>50 \%$ of the lung field within $24-48$ hours. SARS-CoV-2 infection was confirmed by two sequential real-time reverse transcriptase polymerase chain reaction assays (Abbott Molecular Real Time SARS-CoV-2 assay, Abbott Molecular, Des Plaines, IL, USA) from nasopharyngeal, oropharyngeal and bronchoalveolar lavage fluid swab specimens. Age, medical history, and prior medications were obtained 
from medical records. The attending physicians reported the outcome of the ICU patients as either dead or discharge. Uninfected volunteers were recruited from the Dammam and Qatif areas to serve as controls. All assays were performed in accordance with guidelines for handling and assaying blood samples from COVID-19 patients by Imam Abdulrahman Bin Faisal University.

\section{Blood sampling}

Two blood samples were obtained by a registered nurse from COVID-19 infected patients the morning after admittance (8:00 and 9:30 am) and from uninfected patients upon arrival (also between 8:00 - 9:30 am) in EDTA anticoagulant tubes. One sample of blood from each patient was sent to the hospital laboratory for measurements of glucose, hemoglobin, hematocrit, white blood cells, red blood cells, lymphocytes, platelets sodium, potassium, chloride, calcium, magnesium, albumin, urea, creatinine, total bilirubin, alanine aminotransferase, aspartate aminotransferase, creatine kinase, creatinine kinasemyocardial band, prothrombin time, activated partial thromboplastin time, international normalized ratio, lactate, ferritin, C-reactive protein, total cholesterol, triglyceride, high density lipoprotein, and low density lipoprotein. The second blood sample from each patient was centrifuged at $3500 \mathrm{rpm}$ for 5 minutes at $4^{\circ} \mathrm{C}$ in a refrigerated centrifuge and plasma was collected, aliquoted and stored at $-80^{\circ} \mathrm{C}$ for measurements of the following below biomarkers.

\section{Methylglyoxal (HSA-MG equivalent) in plasma}

Levels of human serum albumin-methylglyoxal adduct were measured in plasma samples using the competitive methylglyoxal (MG) ELISA kit based on the inhibition principle. In brief, plasma samples were thawed at room temperature and vortexed $30 \mathrm{sec}$. Different standard concentrations and diluted plasma (1:5) were preincubated with labeled anti-MG trace antibody in U-shaped microtiter plate at room temperature. After 1 hour, $100 \mu \mathrm{L}$ of standard or samples mixed with tracer from the U-shaped plate were transferred in duplicate into appropriate wells in the coated microtiter plate coated with MG-adduct and incubated for 1 hour at room temperature. After three washing, diluted streptavidin- Horseradish peroxidase (HRP) was added to each well and plate was incubated for $60 \mathrm{~min}$ at room temperature. Plate was washed three time and chromogenic substrate 3,3,5, 5-tetramethylbenzidine (TMB) which catalyzes by HRP to generate a blue color was added. After $30 \mathrm{~min}$, a100 $\mu \mathrm{L}$ of acidic stop solution was added into each well and the absorbance at $450 \mathrm{~nm}$ was recorded using a using a Biotek Synergy Neo2 HTS MultiMode Microplate Reader (Männedorf, Switzerland). A standard curve was generated, and MG-adduct concentrations were calculated.

\section{Glyoxlase-1 (Glo1) in plasma}

Glyoxalase-I (Glo1) levels in plasma from uninfected and COVID-19 were detected using commercial ELISA kits according to the manufacturer's protocols (catalog\# MBS2021816, MyBioSource, Inc., San Diego, CA, USA). Briefly, 100- $\mu \mathrm{L}$ of different concentration standards or diluted plasma samples (1:10) were added to the wells in duplicate, and the plate was incubated for $60 \mathrm{~min}$ at $37^{\circ} \mathrm{C}$. After this, $100-\mu \mathrm{L}$ of detection reagent $A$ was added to each well, and the plate was incubated for $60 \mathrm{~min}$ at $37^{\circ} \mathrm{C}$. Thereafter, the plate was washed three times and a $100-\mu \mathrm{L}$ of detection reagent $\mathrm{B}$ working solution was added to 
each well, and the plate was incubated for $30 \mathrm{~min}$ at $37^{\circ} \mathrm{C}$. After 5 washes, $90-\mu \mathrm{L}$ of TMB substrate solution was added into each well, incubated for 20 minutes at $37^{\circ} \mathrm{C}$. Finally, 50 -ul of stop solution was loaded into each well and the absorbance was measured at $450 \mathrm{~nm}$ using the Biotek Synergy Neo2 HTS Multi-Mode Microplate Reader. The intensity of the color product was directly proportional to concentration of Glo-1 in plasma.

\section{Glutathione in plasma}

The total glutathione concentration in plasma was determined using the glutathione assay Kit (catalog\#. CS0260; Sigma-Aldrich, Inc., St Louis, MO, USA). First, the plasma samples were deproteinized with equal volume of the $5 \% 5$-sulfosalicylic acid solution, vortexed vigorously for about $30 \mathrm{sec}$, incubated for 10 min at $4^{\circ} \mathrm{C}$, centrifuged at $10,000 \times \mathrm{g}$ for 10 minutes at $4^{\circ} \mathrm{C}$ and supernatants were collected. $\mathrm{A}$ glutathione standard curve from 1.56 to 50 nanomoles was prepared. To each well of a 96-well plate, 150 $\mu \mathrm{L}$ of working mixture and a $10 \mu \mathrm{L}$ of standard or $100 \mu \mathrm{L}$ of plasma were added in duplicate. Plate was incubated for $5 \mathrm{~min}$ at room temperature and then $50 \mu \mathrm{L}$ of nicotinamide adenine dinucleotide phosphate (NADPH) solution $(0.16 \mathrm{mg} / \mathrm{mL})$ was added. The absorbance at $412 \mathrm{~nm}$ was recorded using a microplate reader. A standard curve was generated by linear regression and glutathione concentrations were calculated.

\section{Tumor necrosis factor alpha (TNF-a) in plasma}

Tumor necrosis factor alpha (TNF-a) level in plasma was measured using an ELISA kit (catalog\#. ab181421, Abcam Inc, Cambridge, MA, USA) according to the manufacturer's instruction. Briefly, a 50- $\mu \mathrm{L}$ of standard or $50-\mu \mathrm{L}$ of undiluted tested samples were added to the wells in duplicate and then a $50-\mu \mathrm{L}$ of antibody cocktail (a mixture of capture and detector antibody) was added to each well. The plate was incubated for 1 hour at room temperature on a plate shaker stetted to $400 \mathrm{rpm}$. After 3 washing, 100- $\mu \mathrm{L}$ of TMB substrate was loaded and incubated for 10 minutes in the dark on a plate shaker stetted to 400 rpm. Finally, $100-\mu \mathrm{L}$ of stop solution was added to each well and the optical density (OD) was recoded at $450 \mathrm{~nm}$ using a Biotek ELX 800 microplate reader.

\section{Interleukin-1 beta (IL-1b) levels in plasma}

Plasma level of human interleukin-1 beta (IL-1 $\beta$ ) was measured according to the manufacturer's protocols (ELISA kit, Cat \# ab 46052, Abcam Inc, Cambridge, MA, USA). A 100- $\mu \mathrm{L}$ of standard, control and tested samples in duplicate were added to the wells. Biotinylated anti-IL-1b was then added, and the plate was incubated for 3 hours at room temperature. After 3 washings, $100-\mu \mathrm{L}$ of streptavidin- HRP solution was added into all wells and incubated for 30 minutes. Next, plate was washed 3 times and each well was incubated with $100 \mu \mathrm{L}$ of TMB in the dark for 15 minutes at room temperature followed by $100-\mu \mathrm{L}$ of acidic stop solution. The absorbance at $450 \mathrm{~nm}$ was recorded using the microplate reader.

\section{Semicarbazide-sensitive amine oxidase (SSAO) activity in plasma}


The plasma levels of semicarbazide-sensitive amine oxidase (SSAO) were determined using the FluoroSSAO assay Kit (catalog\# SSA0100-3; Cell Technology, Inc. 23575 Cabot Blvd., Suite 203. Hayward, CA 94545 USA). In this experiment, all plasma tested samples were diluted in ratio of 1:5 by reaction buffer. Since benzylamine served as a substrate for both SSAO and monoamine oxidase B, pargyline, a monoamine oxidase $B$ inhibitor, was then added to a final concentration of $0.5 \mathrm{mM}$ to each sample and incubated for 30 minutes at $37^{\circ} \mathrm{C}$. In a black 96 -well plate, $100 \mu \mathrm{L}$ of standard or sample were added to each individual wells. Thereafter, $100 \mu \mathrm{L}$ of the reaction cocktail, a mixture of detection reagent, HRP and benzylamine were added to each well and incubated at $37^{\circ} \mathrm{C}$ for 2 hours. After 2 hours, the plate was read with excitation at 530-570 nm and emission at $590 \mathrm{~nm}$ using Biotek Synergy Neo2 HTS Multi-Mode Microplate Reader.

\section{Statistical Analyses}

Data were analyzed using GraphPad Prism 7.0 software (La Jolla, CA) and Statistical Package for Social Sciences (SPSS) version 26.0. Armonk, NY: IBM Corp, and presented in text as mean \pm standard error of the mean (SEM). T-test for independent samples or one-way ANOVA with Brown-Forsythe and Bartlett tests were used for continuous data. Logistic regression analysis was also used to explore the influence of the examined variables on death/survival. Significant differences were considered at $P<0.05$.

\section{Declarations}

\section{Author Contributions}

FAA and KRB conceived the experiments and planned along with all other authors. FAA, MNA, SA, MFA, AKA, AA, HA, SRB, MAM, AJA, HA and KRB conducted experiments and performed data analyses. SA, MNA, H.M.A, MA, AKA, FA, MJA, MSA, FSA, ASA, HS, RAA, MHA, and MSA collected and analyzed patient profile data. MA, AA, and SA were responsible for ICU COVID-19 and recruitment of uninfected controls. KRB, FAA, and SA interpreted the data, wrote, and edited the manuscript.

\section{Conflicts of Interest}

The authors declare no conflict of interest.

\section{Data Availability}

Data are available from the corresponding author upon reasonable request. FAA and KRB is the guarantors of this work, has full access to all the data in the study and takes responsibility for the integrity of the data and the accuracy of the data analyses.

\section{Acknowledgements}

This work is primarily supported by a grant from the King Abdulaziz City for Science and Technology (KACST \# 0007-070-01-20-5) and the Deanship of Scientific Research, Imam Abdulrahman Bin Faisal University (Covid19-2020-012-Med). 
The authors would like to express their appreciation to KACST for their continuing support throughout our work. Very special thanks to Prof. Fahad Ahmed Al-Harbi. Without his endless support, encouragement, and suggestions, we could have never started and completed this work. We also thank to Husain AlSuwaileh and Jaffar Alsaddah at Qatif Hospital, for facilitating some of the lab work.

\section{References}

1. Weidmann, M.D., Ofori, K. \& Rai, A.J. Laboratory Biomarkers in the Management of Patients With COVID-19. Am J Clin Pathol 155, 333-342 (2021).

2. Johns Hopkins University and Medicine (2021). Coronavirus COVID-19 Global Cases by the Center for Systems Science and Engineering (CSSE) (2021).

3. Pierron, D. et al. Smell and taste changes are early indicators of the COVID-19 pandemic and political decision effectiveness. Nat Commun 11, 5152 (2020).

4. Du, R.H. et al. Hospitalization and Critical Care of 109 Decedents with COVID-19 Pneumonia in Wuhan, China. Ann Am Thorac Soc 17, 839-846 (2020).

5. Younis, N.K., Zareef, R.O., Maktabi, M.A.N. \& Mahfouz, R. The Era of the Coronavirus Disease 2019 Pandemic: A Review on Dynamics, Clinical Symptoms and Complications, Diagnosis, and Treatment. Genet Test Mol Biomarkers 25, 85-101 (2021).

6. Amdal, C.D. et al. Health-related quality of life issues, including symptoms, in patients with active COVID-19 or post COVID-19; a systematic literature review. Qual Life Res (2021).

7. Williamson, E.J. et al. Factors associated with COVID-19-related death using OpenSAFELY. Nature 584, 430-436 (2020).

8. Velavan, T.P. et al. Host genetic factors determining COVID-19 susceptibility and severity. EBioMedicine 72, 103629 (2021).

9. Venkatakrishnan, A.J. et al. Mapping each pre-existing condition's association to short-term and long-term COVID-19 complications. NPJ Digit Med 4, 117 (2021).

10. Moghadas, S.M. et al. The impact of vaccination on COVID-19 outbreaks in the United States. Clin Infect Dis (2021).

11. Barber, T.M. COVID-19 and diabetes mellitus: implications for prognosis and clinical management. Expert Rev Endocrinol Metab 15, 227-236 (2020).

12. Al-Sabah, S., Al-Haddad, M., Al-Youha, S., Jamal, M. \& Almazeedi, S. COVID-19: Impact of obesity and diabetes on disease severity. Clin Obes 10, e12414 (2020). 
13. Halvatsiotis, P. et al. Demographic and clinical features of critically ill patients with COVID-19 in Greece: The burden of diabetes and obesity. Diabetes Res Clin Pract 166, 108331 (2020).

14. Aiyegbusi, O.L. et al. Symptoms, complications and management of long COVID: a review. J R Soc Med 114, 428-442 (2021).

15. Elhiny, R., Al-Jumaili, A.A. \& Yawuz, M.J. An overview of post-COVID-19 complications. Int J Clin Pract 75, e14614 (2021).

16. Samprathi, M. \& Jayashree, M. Biomarkers in COVID-19: An Up-To-Date Review. Front Pediatr 8, 607647 (2020).

17. Ponti, G., Maccaferri, M., Ruini, C., Tomasi, A. \& Ozben, T. Biomarkers associated with COVID-19 disease progression. Crit Rev Clin Lab Sci 57, 389-399 (2020).

18. Topp, G. et al. Biomarkers Predictive of Extubation and Survival of COVID-19 Patients. Cureus 13, e15462 (2021).

19. Malik, P. et al. Biomarkers and outcomes of COVID-19 hospitalisations: systematic review and meta-analysis. BMJ Evid Based Med 26, 107-108 (2021).

20. Narvel, H., Sayed, A., Narvel, N., Yakkali, S. \& Katchi, T. Do Certain Biomarkers Predict Adverse Outcomes in Coronavirus Disease 2019? J Clin Med Res 13, 195-203 (2021).

21. Li, M.Y., Li, L., Zhang, Y. \& Wang, X.S. Expression of the SARS-CoV-2 cell receptor gene ACE2 in a wide variety of human tissues. Infect Dis Poverty 9, 45 (2020).

22. Song, J. et al. Systematic analysis of ACE2 and TMPRSS2 expression in salivary glands reveals underlying transmission mechanism caused by SARS-CoV-2. J Med Virol 92, 2556-2566 (2020).

23. Tumpara, S. et al. Boosted Pro-Inflammatory Activity in Human PBMCs by Lipopolysaccharide and SARS-CoV-2 Spike Protein Is Regulated by alpha-1 Antitrypsin. Int J Mol Sci 22(2021).

24. Mansouri, K. et al. Can a metabolism-targeted therapeutic intervention successfully subjugate SARS-COV-2? A scientific rational. Biomed Pharmacother 131, 110694 (2020).

25. Codo, A.C. et al. Elevated Glucose Levels Favor SARS-CoV-2 Infection and Monocyte Response through a HIF-1alpha/Glycolysis-Dependent Axis. Cell Metab 32, 498-499 (2020).

26. Krishnan, S. et al. Metabolic perturbation associated with COVID-19 disease severity and SARSCoV-2 replication. Mol Cell Proteomics, 100159 (2021).

27. Ajaz, S. et al. Mitochondrial metabolic manipulation by SARS-CoV-2 in peripheral blood mononuclear cells of patients with COVID-19. Am J Physiol Cell Physio/ 320, C57-C65 (2021). 
28. Moolamalla, S.T.R., Balasubramanian, R., Chauhan, R., Priyakumar, U.D. \& Vinod, P.K. Host metabolic reprogramming in response to SARS-CoV-2 infection: A systems biology approach. Microb Pathog 158, 105114 (2021).

29. Gibellini, L. et al. Altered bioenergetics and mitochondrial dysfunction of monocytes in patients with COVID-19 pneumonia. EMBO Mol Med 12, e13001 (2020).

30. Singh, K. et al. Network Analysis and Transcriptome Profiling Identify Autophagic and Mitochondrial Dysfunctions in SARS-CoV-2 Infection. bioRxiv (2020).

31. Richard, J.P. Mechanism for the formation of methylglyoxal from triosephosphates. Biochem Soc Trans 21, 549-53 (1993).

32. Thornalley, P.J. The glyoxalase system in health and disease. Mol Aspects Med 14, 287-371 (1993).

33. Sousa Silva, M., Gomes, R.A., Ferreira, A.E., Ponces Freire, A. \& Cordeiro, C. The glyoxalase pathway: the first hundred years... and beyond. Biochem J 453, 1-15 (2013).

34. Antognelli, C., Palumbo, I., Aristei, C. \& Talesa, V.N. Glyoxalase I inhibition induces apoptosis in irradiated MCF-7 cells via a novel mechanism involving Hsp27, p53 and NF-kappaB. Br J Cancer 111, 395-406 (2014).

35. Ranganathan, S., Ciaccio, P.J., Walsh, E.S. \& Tew, K.D. Genomic sequence of human glyoxalase-l: analysis of promoter activity and its regulation. Gene 240, 149-55 (1999).

36. Schalkwijk, C.G. \& Stehouwer, C.D.A. Methylglyoxal, a Highly Reactive Dicarbonyl Compound, in Diabetes, Its Vascular Complications, and Other Age-Related Diseases. Physiol Rev 100, 407-461 (2020).

37. Schofield, C.J. \& Ratcliffe, P.J. Oxygen sensing by HIF hydroxylases. Nat Rev Mol Cell Biol 5, 34354 (2004).

38. Alomar, F. et al. Smooth muscle-generated methylglyoxal impairs endothelial cell-mediated vasodilatation of cerebral microvessels in type 1 diabetic rats. Br J Pharmacol 173, 3307-3326 (2016).

39. Alomar, F.A. et al. Adeno-Associated Viral Transfer of Glyoxalase-1 Blunts Carbonyl and Oxidative Stresses in Hearts of Type 1 Diabetic Rats. Antioxidants (Basel) 9(2020).

40. Arriagada-Petersen, C. et al. Effect of advanced glycation end products on platelet activation and aggregation: a comparative study of the role of glyoxal and methylglyoxal. Platelets 32, 507-515 (2021).

41. Gawlowski, T., Stratmann, B., Stirban, A.O., Negrean, M. \& Tschoepe, D. AGEs and methylglyoxal induce apoptosis and expression of Mac-1 on neutrophils resulting in platelet-neutrophil aggregation. Thromb Res 121, 117-26 (2007). 
42. Lin, C.C. et al. Methylglyoxal activates NF-kappaB nuclear translocation and induces COX-2 expression via a p38-dependent pathway in synovial cells. Life Sci 149, 25-33 (2016).

43. Masih, A. et al. Discovery of novel pyrazole derivatives as a potent anti-inflammatory agent in RAW264.7 cells via inhibition of NF-kB for possible benefit against SARS-CoV-2. J Biochem Mol Toxicol 35, e22656 (2021).

44. Oliveira, E. et al. ICU outcomes and survival in patients with severe COVID-19 in the largest health care system in central Florida. PLoS One 16, e0249038 (2021).

45. Salmi, M. \& Jalkanen, S. VAP-1: an adhesin and an enzyme. Trends Immunol 22, 211-6 (2001).

46. Hishida, E. et al. Crucial Role of NLRP3 Inflammasome in the Development of Peritoneal Dialysisrelated Peritoneal Fibrosis. Sci Rep 9, 10363 (2019).

47. Black, S., Kushner, I. \& Samols, D. C-reactive Protein. J Biol Chem 279, 48487-90 (2004).

48. Horowitz, R.I., Freeman, P.R. \& Bruzzese, J. Efficacy of glutathione therapy in relieving dyspnea associated with COVID-19 pneumonia: A report of 2 cases. Respir Med Case Rep 30, 101063 (2020).

49. Carlberg, I. \& Mannervik, B. Glutathione reductase. Methods Enzymol 113, 484-90 (1985).

50. Seelig, G.F. \& Meister, A. Glutathione biosynthesis; gamma-glutamylcysteine synthetase from rat kidney. Methods Enzymo/ 113, 379-90 (1985).

51. Su, C.M., Wang, L. \& Yoo, D. Activation of NF-kappaB and induction of proinflammatory cytokine expressions mediated by ORF7a protein of SARS-CoV-2. Sci Rep 11, 13464 (2021).

52. Wang, G.L., Jiang, B.H., Rue, E.A. \& Semenza, G.L. Hypoxia-inducible factor 1 is a basic-helix-loophelix-PAS heterodimer regulated by cellular 02 tension. Proc Natl Acad Sci U S A 92, 5510-4 (1995).

53. Wang, G.L. \& Semenza, G.L. Purification and characterization of hypoxia-inducible factor 1. J Biol Chem 270, 1230-7 (1995).

\section{Figures}

\section{Figure 1}

Plasma levels of MG, glutathione, Glo1 SSAO, TNF-a, IL-1 $\beta$ and CRP in uninfected control and ICU COVID19 patients. Panel A shows that MG levels (detected as its surrogate, HSA-MG) were significantly higher in ICU COVID-19 patients compared to uninfected controls. Panel B shows glutathione levels were significantly lower in ICU COVID-19 patients compared to uninfected controls. Panel C shows that Glo1 
levels in ICU COVID-19 patients were not significantly different from uninfected controls. Panel D shows that SSAO levels were significantly higher in ICU COVID-19 patients compared to uninfected controls. Panel D shows that TNF-a levels were significantly higher in ICU COVID-19 patients compared to uninfected controls. Panel E shows that IL-1 $\beta$ levels were significantly higher in ICU COVID-19 patients compared to uninfected controls. Panel $F$ shows that CRP levels were significantly higher in ICU COVID-19 patients compared to uninfected controls. Data shown are for each patient with mean \pm S.E.M from $n=30$ uninfected controls ( $26.6 \%$ females) and $n=65$ ICU COVID-19 patients $(29.3 \%$ females). Statistical significances are shown above sets of data points on the graphs.

\section{Figure 2}

Plasma levels of MG, glutathione, Glo1, SSAO, TNF-a, IL-1 $\beta$ and CRP in uninfected controls and ICU COVID-19 patients that survived and died. Panel A shows MG in plasma from ICU COVID-19 patients that survived and died were significantly higher than that in uninfected controls. MG levels in plasma from ICU COVID-19 that died was also significantly higher than ICU-COVID-19 patients that survived. Panel B shows glutathione in plasma from ICU COVID-19 patients that survived and died were significantly lower than that in uninfected controls. Glutathione levels in plasma from ICU COVID-19 that died was also significantly lower than ICU-COVID-19 patients that survived. Panel C shows Glo1 in plasma from ICU COVID-19 patients that survived a were not significantly different from than that in uninfected controls. Glo1 in plasma from ICU COVID-19 patients that died was significantly lower than that in in uninfected controls and ICU COVID-19 patients that survived. Panel D shows SSAO activities in plasma from ICU COVID-19 patients that survived and died were significantly higher than that in uninfected controls. However, there were no significant difference in plasma SSAO activities between ICU patients that survived and died. Panel E shows TNF-a in plasma from ICU COVID-19 patients that survived and died were significantly higher than that in uninfected controls. However, there were no significant difference in plasma levels of TNF-a in ICU patients that survived and died. Panel F shows IL-1 $\beta$ in plasma from ICU COVID-19 patients that survived and died were significantly higher than that in uninfected controls. However, there were no significant difference in plasma levels of IL-1 $\beta$ in ICU patients that survived and died. Panel G shows CRP in plasma from ICU COVID-19 patients that survived and died were significantly higher than that in uninfected controls. However, there were no significant difference in plasma levels of CRP in ICU patients that survived and died. Glo1 in plasma from ICU COVID-19 patients that died was significantly lower than that in in uninfected controls and ICU COVID-19 patients that survived. Data shown are mean \pm S.E.M from $n=30$ in uninfected controls ( $26.6 \%$ females), $n=33$ died ( $42.4 \%$ females) and $n=32$ in survived ( $37.5 \%$ females). Statistical significance levels are shown above data points on each graph.

\section{Figure 3}


Plasma levels of MG, glutathione, and in ICU COVID-19 patients without diabetes mellitus (non-DM) and with diabetes (DM) and separated into those that survived and those that died. Panel A shows MG levels were significantly higher in non-DM and DM ICU COVID-19 patients compared to uninfected controls. There were no significant differences in MG levels in non-DM and DM ICU COVID-19 patients. Panel B shows plasma of MG in non-DM and DM ICU COVID-19 patients that died were significantly higher than that in survivors. Panel $\mathrm{C}$ shows glutathione levels were significantly higher in non-DM and DM ICU COVID-19 patients compared to uninfected controls. There were no significant differences in glutathione levels in non-DM and DM ICU COVID-19 patients. Panel D shows plasma of glutathione levels in non-DM and DM ICU COVID-19 patients that died had significantly lower than that in patients that survived. Panel E shows plasma show Glo1 levels in plasma of non-DM and DM patients were not significantly different from that of uninfected controls. Panel F shows plasma of Glo1 levels in non-DM and DM ICU COVID-19 patients that died had significantly lower than that in patients that survived. Data shown in Panels $A, C$ and $E$ are mean \pm S.E.M from each of the $n=30$ uninfected controls $(26.6 \%$ females $), n=34$ for non-DM (33.3\% females) and $n=31$ for DM (25.8\% females). Data shown in Panels B, D and F are mean \pm S.E.M for each of the $n=30$ in uninfected controls ( $26.6 \%$ females), $n=18$ in non-DM survived ( $44.4 \%$ females), $\mathrm{n}=16$ in non-DM died (30.7\% females), $\mathrm{n}=14$ DM in survived ( $28.6 \%$ females), and $n=17$ in DM died (37.5\% females) groups. Statistical significances are shown above data points on each graph.

\section{Figure 4}

Plasma levels of SSAO, TNF-a, IL-1 $\beta$ and CRP in ICU COVID-19 patients without diabetes mellitus (nonDM) and with diabetes (DM) and separated into those that survived and those that died. Panel A shows SSAO activities in plasma from non-DM and DM ICU COVID-19 patients were significantly higher than uninfected controls. SSAO activity in plasma from non-DM and DM patients were not significantly different. Panel B shows no significant differences in SSAO activities in plasma from non-DM and DM ICU COVID-19 patients that died and survived. Panel $C$ shows no significant differences in TNF-a in plasma from non-DM and DM ICU COVID-19 patients. However, their levels were significantly higher than uninfected controls. Panel D shows no significant differences in plasma levels of TNF-a in non-DM and DM ICU COVID-19 patients that died and survived. Panel E shows IL-1 $\beta$ in plasma of non-DM and DM ICU COVID-19 patients were significantly higher than that in uninfected controls. IL-1 $\beta$ in plasma from ICU COVID-19 with DM was also significantly higher than of ICU-COVID-19 patients without DM. Panel F shows no significant difference in plasma levels of IL-1 $\beta$ in non-DM and DM ICU COVID-19 patients that died and survived. Panel $G$ shows significantly higher CRP in plasma from non-DM and DM ICU COVID-19 patients compared to uninfected controls. CRP in plasma from ICU COVID-19 with DM was not significantly higher than ICU-COVID-19 patients without DM. Panel H shows no significant differences in plasma levels of CRP in non-DM and DM ICU COVID-19 patients that died and survived. Data shown in Panels $A, C$ and $E$ are mean \pm S.E.M from $n=30$ in uninfected controls ( $26.6 \%$ females), $n=34$ in non-DM (33.3\% females) and $n=31$ in DM (25.8\% females) group. Data shown in Panels B, D and F are mean \pm S.E.M from $n=30$ in uninfected controls ( $26.6 \%$ females), $n=18$ in non-DM survived ( $44.4 \%$ females), 
$\mathrm{n}=16$ in non-DM died (30.7\% females), $\mathrm{n}=14 \mathrm{DM}$ in survived (28.6\% females), and $\mathrm{n}=17$ in DM died (37.5\% females) groups. Statistical significances are shown above data points on each graph.

\section{Figure 5}

Panel A shows low MG (<2 times higher than uninfected control), moderate MG (between 2-3 times higher than uninfected controls) and high MG (>4times higher than uninfected controls) in ICU patients that survived and died along with $M G$ in uninfected controls. Data shown are mean \pm S.E.M for each of $n=30$ uninfected controls ( $26.6 \%$ females), $n=11$ survived for low, and $n=20$ survived moderate. $n=13$ died for moderate and $n=21$ died, for high MG. Panel B shows Kaplan-Meier survival curve for all ICU COVID-19 patients that died with moderate MG $(n=13)$ and high MG $(n=21)$. Panel $C$ shows Kaplan-Meier survival curve for non-DM ICU COVID-19 patients that died with moderate $(n=8)$ and high MG $(n=8)$. Panel D shows Kaplan-Meier survival curve for DM ICU COVID-19 patients that died with moderate $(n=5)$ and high MG $(n=12)$.

\section{Figure 6}

Correlations between plasma MG and glutathione, Glo1, SSAO, TNF- $\alpha$, IL-1 $\beta$ and CRP in ICU patients that died. Panels $A$ and $B$ show a strong inverse correlation between $M G$ and glutathione $(r 2=-0.62)$, and $M G$ and Glo1 $(r 2=-0.50)$ in ICU patients that died. Panel $C$ shows strong positive correlation $(r 2=0.52)$ between plasma MG and SSAO activity in ICU patients that died. Panel $D$ shows moderate positive correlation $(r 2=0.41)$ between plasma MG and TNF-a in ICU in patients that died. Panels $E, F$ and $G$ show weak correlation between plasma MG and IL-1 $\beta(r 2=0.25), M G$ and CRP $(r 2=0.26)$ and MG and age $(r 2=20)$ in ICU patients that died. Data in graphs are for $n=33$ patients. 